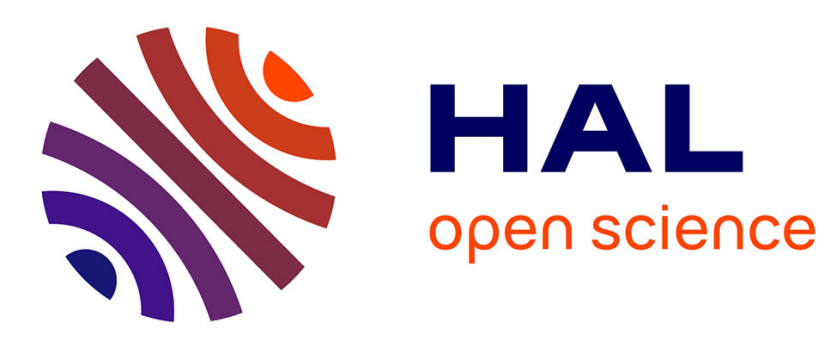

\title{
Flow and air-entrainment around partially submerged vertical cylinders
}

\author{
Valentin Ageorges, Jorge Peixinho, Gaële Perret
}

\section{To cite this version:}

Valentin Ageorges, Jorge Peixinho, Gaële Perret. Flow and air-entrainment around partially submerged vertical cylinders. Physical Review Fluids, 2019, 4 (6), pp.Article number 064801. 10.1103/PhysRevFluids.4.064801 . hal-02292362

\section{HAL Id: hal-02292362 \\ https://hal.science/hal-02292362}

Submitted on 19 Sep 2019

HAL is a multi-disciplinary open access archive for the deposit and dissemination of scientific research documents, whether they are published or not. The documents may come from teaching and research institutions in France or abroad, or from public or private research centers.
L'archive ouverte pluridisciplinaire HAL, est destinée au dépôt et à la diffusion de documents scientifiques de niveau recherche, publiés ou non, émanant des établissements d'enseignement et de recherche français ou étrangers, des laboratoires publics ou privés. 


\title{
Flow and air-entrainment around partially submerged vertical cylinders
}

\author{
Valentin Ageorges, ${ }^{1}$ Jorge Peixinho, ${ }^{1,2}$ and Gaële Perret ${ }^{1}$ \\ ${ }^{1}$ Laboratoire Ondes et Milieux Complexes, CNRS et Université Le Havre Normandie, 76600 Le Havre, France \\ ${ }^{2}$ Laboratoire PIMM, CNRS, Arts et Métiers, CNAM, HÉSAM Université, 75013 Paris, France
}

\begin{abstract}
In this study, a partially submerged vertical cylinder is moved at constant velocity through water, which is initially at rest. During the motion, the wake behind the cylinder induces free-surface deformation. Eleven cylinders, with diameters from $D=1.4$ to $16 \mathrm{~cm}$, were tested under two different conditions: (i) constant immersed height $h$ and (ii) constant $h / D$. The range of translation velocities and diameters are in the regime of turbulent wake with experiments carried out for $4500<\operatorname{Re}<240000$ and $0.2<\mathrm{Fr}<2.4$, where $\mathrm{Re}$ and Fr are the Reynolds and Froude numbers based on $D$. The focus here is on dragforce measurements and relatively strong free-surface deformation up to air-entrainment. Specifically, two modes of air-entraiment have been uncovered: (i) in the cavity along the cylinder wall and (ii) in the wake of the cylinder. A scaling for the critical velocity for air-entrainment in the cavity has been observed in agreement with a simple model. Furthermore, for $\mathrm{Fr}>1.2$, the drag force varies linearly with $\mathrm{Fr}$.
\end{abstract}

\section{INTRODUCTION}

The flow past ships [1,2], or an emerged body such as a bridge pillar, is a fundamental, familiar, and fascinating sight. Measurements and modeling of this simple flow can have relevance for the floating offshore structures and renewable energy systems [3,4]. The disordered motion of the free-surface in the wake of the cylinder is related to the drag and lift effect experienced by the cylinder. The wake pattern is responsible for the surface waves and probably the air-entrainment. Many experiments and numerical works have considered a single vertical cylinder geometry in a fully submerged situation [5-9]. These works reported the drag-force measurements on the circular

cylinders as a function of the Reynolds number $\operatorname{Re}$ defined as $\operatorname{Re}=U D / v$, with $U$ being the velocity, $D$ the cylinder diameter, and $v$ the kinematic viscosity of the fluid. In addition, Schewe [5] showed the occurrence of a drag crisis at $\mathrm{Re} \approx 350000$, which separates two distinct regions of a nearly constant dimensionless drag coefficient, $C_{D}$, defined later. In the subcritical regime, for $\operatorname{Re}<350000$, the drag coefficient and the vortex-shedding frequency $f$, characterized by the Strouhal number $\mathrm{St}=f D / U$, are almost constant at $C_{D} \approx 1.2$ and $\mathrm{St} \approx 0.2$. In this regime, the flow is characterized by a turbulent wake with a laminar boundary layer separation [10].

In contrast to fully submerged situations, few studies have been done taking into account the effect of a free-surface, which modifies the pressure distribution around the cylinder. From a numerical point of view, the authors of Refs. [11-13] have computed the flow around a wallmounted cylinder, the evolution of $C_{D}$, and the free-surface elevation for Re varying from 27000 up to 458000 . They have shown that the vorticity and $C_{D}$ vary with depth along the cylinder and diminish close to the free-surface. Additionally, $C_{D}$ is strongly influenced by free-end effects for finite-height cylinders [14]. Specifically, Gonçalves et al. [15] reported experimental results in a recirculating water flume for cylinders piercing the free-surface with the aspect ratio $0.1<h / D<2$, where $h$ is the immersed cylinder height. They focused on forces and particle image velocimetry measurements for $10000<\operatorname{Re}<50000$ and $0.07<\mathrm{Fr}<0.36$; the Froude number Fr is defined as 


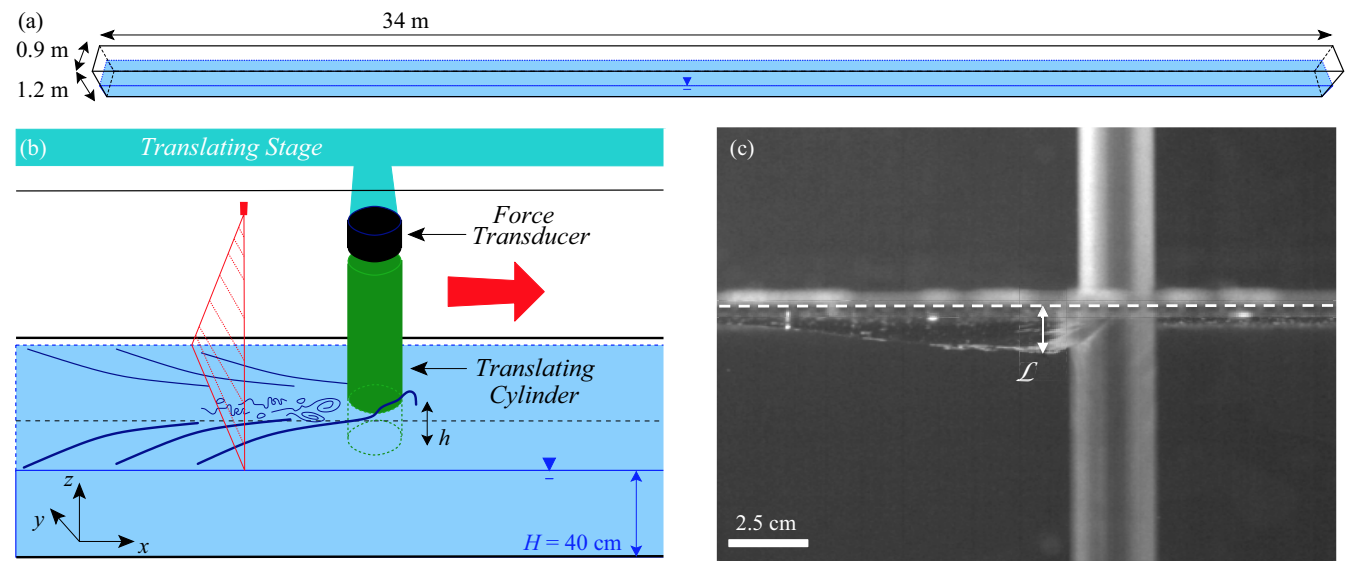

FIG. 1. Experimental setup. (a) Schematic tilted view of the flume (drawn up to scale) with the following dimensions: $34 \times 1.2 \times 0.9 \mathrm{~m}$. (b) Schematic of the arrangement around the cylinder, view from the side abovesurface. The shaded red area represents the laser sheet used for the optical method. (c) Typical picture side view. The cylinder is $2.5 \mathrm{~cm}$ in diameter and has been translated from left to right with a velocity of $45.8 \mathrm{~cm} / \mathrm{s}$, $\operatorname{Re}=11500$, and $\mathrm{Fr}=0.9$. The depth of the cavity $\mathcal{L}$ is represented in the near cylinder wake.

Fr $=U / \sqrt{g D}$, with $g=9.81 \mathrm{~m} / \mathrm{s}^{2}$ being the gravitational acceleration. For constant $\operatorname{Re}, C_{D}$ values increase with $h / D$. Moreover, the velocity fields at $\mathrm{Re}=43000$ revealed two recirculation regions: (i) below the cylinder and (ii) behind the cylinder. The measurements in Chaplin and Teigen [16] also showed that $C_{D}$ values reach a maximum with respect to Fr. The evolution of $C_{D}$ values is in agreement with the experiments of Ducrocq et al. [17], who investigated the behavior of subcritical and supercritical flows for different slopes of a flume with $0.5<\mathrm{Fr}<2.3$ and $\operatorname{Re}=50000$.

In recent years, numerical studies have reproduced the flow and drag around free-end cylinders for different $h / D[12,13,17,18]$, but there is a lack of data for $\operatorname{Re}$ around the drag crisis $(\operatorname{Re} \approx$ 350000 ) and almost no data for strong free-surface deformations and rupture. An important numerical output is the mean interface elevation [12,13]. Specifically, Keough et al. [19] analyzed the evolution of the fountain height ahead of the cylinder for Re varying between 45000 and 380000. They concluded a dependency of the fountain height with $\mathrm{Fr}^{2}$ and revealed that this evolution follows the Bernoulli equation for small velocity. In addition, at the downstream side of the cylinder, the maximum cavity depth $\mathcal{L}$ is also believed to scale with $\operatorname{Fr}^{2}$ [16]. The cavity depth $\mathcal{L}$ is schematized in Fig. 1(c). Depending on the cylinder diameter, the free-surface downstream is locally subjected to strong deformations and air-entrainment in the liquid phase. Benusiglio [20] recently demonstrated a velocity threshold for air-entrainment in the cavity of a translating vertical cylinder. This phenomenon can induce drag reduction effects and it is independent of the drag crisis introduced before. At larger Fr, Hay [21] reported an extensive study of the ventilated regime, where air reaches the bottom of the cylinder and creates a ventilated cavity with large waves and sprays above the nominal free-surface. Modern studies of the artificially or atmospherically ventilated structures (plates or hydrofoils) close to a free-surface are concerned with the stability of ventilated cavities (e.g., Ref. [22]) or properties such as the void fraction measurements [23].

The goal of the present investigation is to quantify the onset of air-entrainment effects. In addition, the present study adds new experimental results and discusses new drag measurements carried out for $4500<\operatorname{Re}<240000$ and $0.2<\mathrm{Fr}<2.4$ with a specific focus on the onset of air-entrainment [22,24-26]. This paper is composed of two parts. In the first part, the experimental setup is described together with an optical method for free-surface height reconstruction. In the second part, results are presented with the drag-force measurements and the determination of the 
TABLE I. Range of Re and Fr numbers for constant $h$ and constant $h / D$ runs.

\begin{tabular}{|c|c|c|c|c|c|c|c|c|c|c|}
\hline \multicolumn{6}{|c|}{$h=23 \pm 0.1 \mathrm{~cm}$} & \multicolumn{5}{|c|}{$h / D=2.55 \pm 0.05$} \\
\hline$D(\mathrm{~cm})$ & $h / D$ & $\mathrm{Re}_{\min }$ & $\mathrm{Re}_{\max }$ & $\mathrm{Fr}_{\min }$ & $\mathrm{Fr}_{\max }$ & $h(\mathrm{~cm})$ & $\mathrm{Re}_{\min }$ & $\mathrm{Re}_{\max }$ & $\mathrm{Fr}_{\min }$ & $\mathrm{Fr}_{\text {max }}$ \\
\hline 1.4 & 16.50 & 7470 & 12460 & 1.45 & 2.41 & 3.6 & 4340 & 12160 & 0.87 & 2.41 \\
\hline 2.5 & 9.20 & 12460 & 24910 & 1.00 & 2.10 & 6.4 & 9730 & 21880 & 0.81 & 1.82 \\
\hline 3 & 7.77 & 17440 & 29900 & 1.08 & 1.84 & 7.6 & 15950 & 27900 & 0.98 & 1.72 \\
\hline 4 & 5.75 & 24900 & 39860 & 1.00 & 1.60 & 10.2 & 19930 & 39860 & 0.80 & 1.60 \\
\hline 5 & 4.60 & 30630 & 59800 & 0.86 & 1.71 & 12.8 & 28460 & 54550 & 0.86 & 1.64 \\
\hline 6 & 3.83 & 40840 & 71460 & 0.87 & 1.52 & 15.3 & 29180 & 68080 & 0.65 & 1.52 \\
\hline 7 & 3.28 & 29900 & 97260 & 0.52 & 1.74 & 17.9 & 32820 & 97260 & 0.58 & 1.72 \\
\hline 8 & 2.87 & 34040 & 122510 & 0.50 & 1.70 & 20.4 & 34040 & 116710 & 0.49 & 1.69 \\
\hline 9 & 2.55 & 34880 & 119600 & 0.41 & 1.42 & 23.0 & 34880 & 119600 & 0.41 & 1.42 \\
\hline 11 & 2.09 & 39860 & 188870 & 0.35 & 1.62 & 28.1 & 39860 & 184370 & 0.35 & 1.62 \\
\hline 16 & 1.44 & 40860 & 239190 & 0.20 & 1.20 & 39.9 & 39860 & 239190 & 0.20 & 1.20 \\
\hline
\end{tabular}

velocity threshold for air-entrainment in the cavity. Finally, the air-entrainment results are compared with a simple model based on the balance between acceleration and gravity.

\section{EXPERIMENTAL SETUP}

The cylinders are hollow and made of polymethyl methacrylate (PMMA) with external diameters of $30,40,50,60,70,80,90,110$, and $160 \mathrm{~mm}$. Their total height is $65 \mathrm{~cm}$ and their wall thickness is $5 \mathrm{~mm}$. The Young modulus of PMMA is $3.1 \mathrm{GPa}$. There are additional hollow cylinders of 14 and $25 \mathrm{~mm}$ made respectively of copper and aluminum. All the cylinders are clogged at their ends. These cylinders are attached vertically, perpendicular to the water free-surface, and translated on a carriage riding horizontally along the flume of $34 \mathrm{~m}$ in length, $90 \mathrm{~cm}$ in width, and $120 \mathrm{~cm}$ in height. The flume is shown schematically in Fig. 1(a). The carriage $(162 \mathrm{~kg})$ is driven by a $600-\mathrm{V}$ ac motor $(34 \mathrm{~kg}$ ), producing a maximum torque of $102 \mathrm{~N} / \mathrm{m}$. A protocol is applied where first the velocity increases linearly along a distance of $3.5 \mathrm{~m}$. Then, the speed is maintained constant over a distance of $9.5 \mathrm{~m}$, where force measurements are acquired in steady state. Finally, the speed decreases linearly over a distance of $2 \mathrm{~m}$.

Two windows are located on the sidewalls of the flume, allowing local flow visualization around the cylinder. The determination of the critical velocity for air-entrainment behind the cylinder is based on images from a $2000 \times 2000$ pixel CCD camera (Basler aca2040) with focal length of $50 \mathrm{~mm}$ placed at $6 \mathrm{~m}$ from the start of the cylinder run. At this position along the flume, the force measurement (described later) has reached a steady-state value. The spatial resolution of the CCD camera is $300 \mu \mathrm{m}$ per pixel and its acquisition frequency is 100 frames per second. Hence, bubbles smaller than $300 \mu \mathrm{m}$ cannot be detected. A typical image of the cylinder passing through the field of view of the camera is shown in Fig. 1(c).

The working fluid is tap water and its height in the flume is constant: $H=40 \pm 0.1 \mathrm{~cm}$. The temperature is monitored; hence the viscosity, the density $\rho$, and the surface tension $\sigma$ of water are corrected to take into account these variations. Indeed between all experimentations, the temperature of water changes from 16.1 to $21.3^{\circ} \mathrm{C}$. In this range of temperature, density variations represent $0.1 \%$ [27], while viscosity variations represent $13.3 \%$ [28]. The correction allows us to keep the error for Re between 0.8 and 9.8\%. The Bond number [29], Bo $=\rho g D^{2} / \sigma$, varies between 26 and 3450 , which implies that the capillary effects are weak.

Two series of experiments were performed: (i) at constant immersion depth $h=23 \pm 0.1 \mathrm{~cm}$ leading to $h / D$ ratios varying between 1.44 and 16.5 and (ii) at constant ratio $h / D=2.55 \pm 0.05$. In total, 372 runs were performed, as summarized in Table I, covering a wide range of Re and Fr. The maximum blockage width of the experiment, $D / W$, where $W$ is the flume width, is 0.177 , 
a value below the critical blockage width of 0.2 for the confinement effect on three-dimensional dynamics proposed by Griffith et al. [30]. In addition, the International Towing Tank Conference (ITTC) 2002 [31] requires a vertical confinement, $(H-h) / h>0.8$. For the current experiments, at constant $h$, the vertical confinement is 0.74 , and for constant $h / D$, this criteria is satisfied for $D<9 \mathrm{~cm}$.

\section{A. Force measurements}

A piezoelectric sensor (Kistler 9327C) placed above the cylinder [see drawing in Fig. 1(b)] measures the axial force $F_{x}$ acting on the whole cylinder with a sensitivity of $-7.8 \mathrm{pC} / \mathrm{N}$. The sensor is connected to a charge amplifier equipped with a low-pass filter of $30 \mathrm{~Hz}$. The contribution of air drag is neglected. Indeed, when $h$ is minimum, the maximum error on $C_{D}$ is about $0.8 \%$. Force signals are filtered using a moving average on 20 points. In order to measure the effect of air-entrainment on drag forces, a time average is performed on $F_{x}$ during $5 \mathrm{~s}$ at constant translating velocity. It is denoted $\bar{F}_{x}$. The drag coefficient $C_{D}$ can thus be defined as

$$
C_{D}(t)=\frac{2 \bar{F}_{x}}{\rho h D U^{2}}
$$

The Strouhal number, defined earlier, is found to be close to $0.2 \pm 0.025$ for $D<6 \mathrm{~cm}$, using the peak of $f$ from the spectrum of lift-force signal. However, for larger diameters, the signal of lift force is noisy, presumably due to the turbulent flow or vibrations of the carriage. Moreover, the range of Re studied here and the materials used here suggest natural frequencies which are well separated from those associated with vortex-induced vibration, which is the motion induced on the elastic cylinder interacting with the turbulent fluid flow. Such vortex-induced vibration phenomena of the elastic cylinder deformation would act like an oscillator inducing several modes corresponding to different vortex sheddings observed by several authors [32-35].

\section{B. Optical method}

The parameter that needs to be measured is the elevation of the free-surface. The height at rest $H$ is well controlled. However, when the cylinder passes through the observation area, the light from a vertical laser sheet located above the flume and pointing to the free-surface, represented in red in Fig. 1(b), is shifted vertically from its initial position. The shift is the difference between the interface at rest and the deformed free-surface. It is proportional to the elevation of the free-surface. The images are captured by a camera placed on the top of the flume with a low incident angle to the free-surface. The proportionality is calibrated by recording images of the laser sheet at five known positions over $25 \mathrm{~mm}$. This technique is able to detect free-surface heights with a precision estimated to $\pm 0.1 \mathrm{~mm}$.

Figure 2 presents examples of free-surface reconstructions using the optical method for $\mathrm{Re}=$ 8500. Two Fr are shown: (a) $\mathrm{Fr}=0.5$ and (b) $\mathrm{Fr}=1.6$. When the Fr increases, the free-surface experiences elevations of larger amplitude. The diagrams allow one to distinguish the fountain height upstream of the cylinders. It is also possible to see the angle of the V-shaped wakes. For $\mathrm{Fr}=1.6$ the $\mathrm{V}$-shaped wake induces a planar wave downstream of the cylinder. Note that this method and other optical methods (e.g., Ref. [36]) are restricted by the dispersion of the laser due to the cylinder's turbulent wake. Hence, the present technique is limited for small Fr and Re.

\section{RESULTS AND DISCUSSION}

The results consist of drag measurements, surface elevation measurements, and image analysis. These have been obtained for different cylinder diameters translated at various speeds with constant immersion $h$ and constant ratio $h / D$. The different modes of air-entrainment are depicted and used 


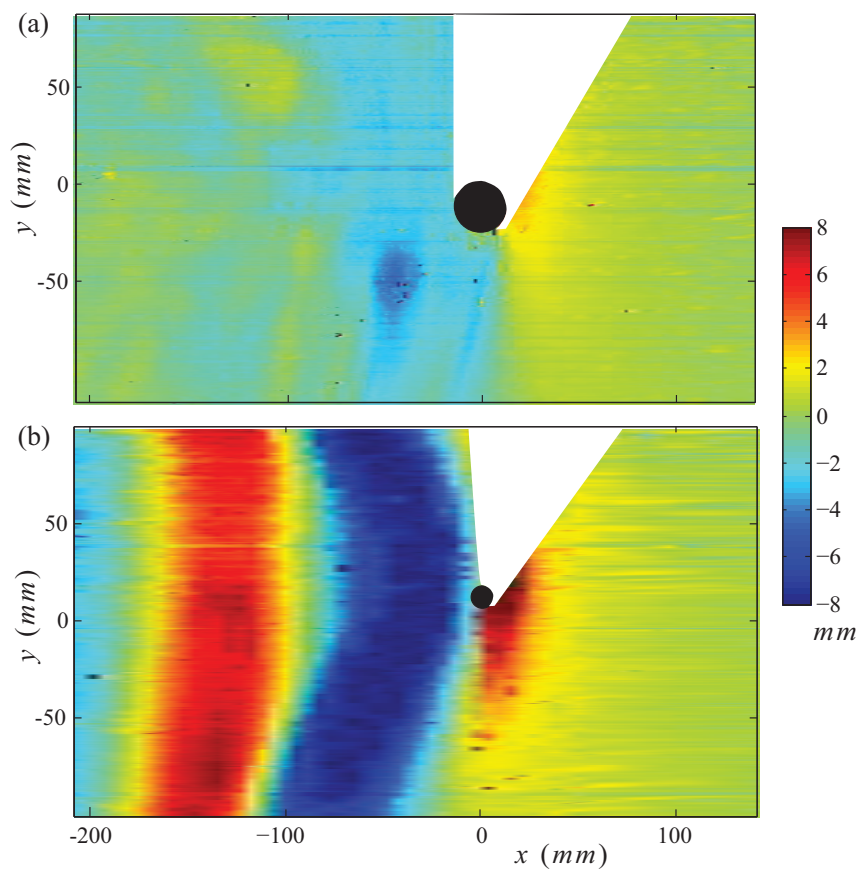

FIG. 2. Free-surface height reconstruction for $\operatorname{Re}=8500$. (a) $D=3 \mathrm{~cm}$ and $\mathrm{Fr}=0.5$. (b) $D=1.4 \mathrm{~cm}$ and $\mathrm{Fr}=1.6$. The cylinders are indicated by black circles and the white bands correspond to shadow areas of the cylinders that cannot be analyzed.

to detect the critical velocity for air-entrainment in the cavity. The scaling of the velocity can be explained using a simple model described below.

\section{A. Drag measurements}

In this section, force measurements are reported in a range of Re and Fr, such that the transition to air-entrainment is observed. Figure 3 presents drag coefficient measurements for different cylinder diameters as a function of $\mathrm{Re}$, at constant $h=23 \pm 0.1 \mathrm{~cm}$ and at constant ratio $h / D=2.55 \pm 0.05$. Solid (empty) symbols correspond to cases without (with) air-entrainment in the cavity $\mathcal{L}$ behind the cylinder. The error bars correspond to the standard deviation of $C_{D}$. The results obtained are compared with the results of Schewe [5] for single-phase flow. Additional results of Refs. [12,15] on surface-piercing cylinders are represented for the same range of Re and $h / D$. In accordance with previous studies, the drag coefficient is found to be about $30 \%$ smaller for surface-piercing cylinders than for single-phase flows. The numerical results of $\mathrm{Yu}$ et al. [12] at three different Re numbers and a constant ratio $h / D=4$ are in good agreement with the present measurements. The results of Gonçalves et al. [15] for a ratio of $h / D=2$ show a nearly constant drag coefficient at higher values than the present study. In their experiments the cylinder is attached to the walls of the flume and the aspect ratio $h / D$ is varied by varying the water depth. Moreover, their maximum Fr based on the cylinder diameter is $\mathrm{Fr}=0.36$, lower than the present values and below the threshold of air-entrainment. This may explain their highest $C_{D}$ values.

In the present study, the drag coefficient first increases with the Re and then decreases after air-entrainment has occurred in the cavity downstream from the cylinder. Air-entrainment is defined when the first bubble is observed or monitored from the camera images (i.e., bubble larger than $300 \mu \mathrm{m})$. In Fig. 3, for small $h / D$ or large diameters $D, C_{D}$ values first increase with $\operatorname{Re}$ after the appearance of the first air bubbles. Then, $C_{D}$ values decrease from $10 \%$ for the 4-cm-diameter 

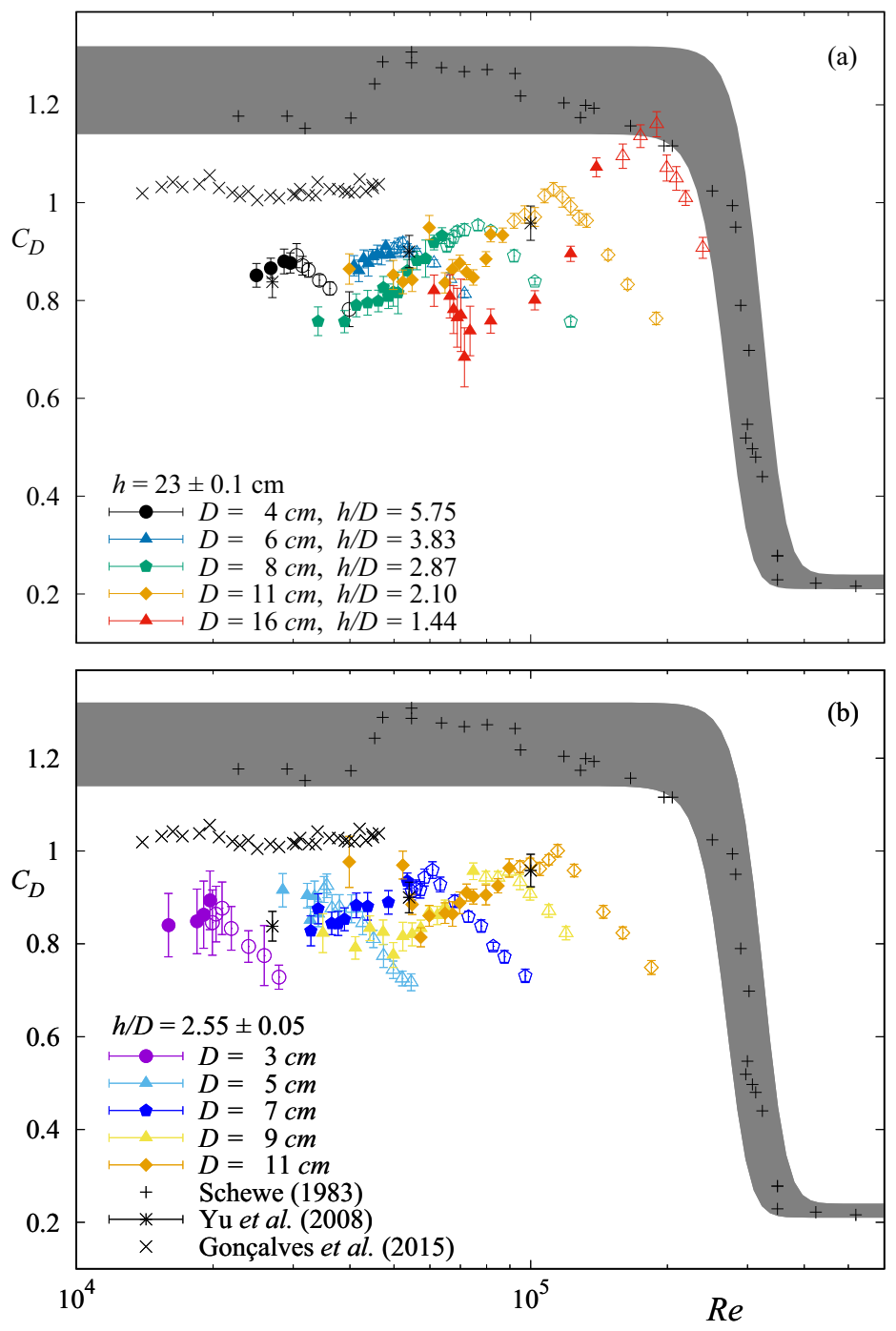

FIG. 3. Drag coefficients, $C_{D}$, as a function of the Reynolds number for different cylinder diameters $D$. (a) Constant immersion depth $h=23 \pm 0.1 \mathrm{~cm}$ and (b) constant ratio $h / D=2.55 \pm 0.05$. Solid symbols represent the situation without air-entrainment and empty symbols represent flow with air-entrainment behind the cylinder. Additional results from the literature are added and the results without free-surface are highlighted using a gray band.

cylinder to $26 \%$ at maximum for the $11-\mathrm{cm}$-diameter cylinder. The existence of the drag reduction effect could be explained by the modification of pressure distribution along the cylinder.

In order to study the influence of the gravity effect, the drag coefficient $C_{D}$ is also plotted as a function of Fr for a constant immersion depth, $h=23 \pm 0.1 \mathrm{~cm}$, in Fig. 4(a) and for a constant ratio, $h / D=2.55 \pm 0.05$, in Fig. 4(b). As in Fig. 3, solid (empty) symbols correspond to measurements without (with) air-entrainment in the cavity. In both cases, the evolution of the drag coefficient clearly shows a transition occurring at $\mathrm{Fr} \approx 1.2$. For $\mathrm{Fr}<1.2, C_{D}$ is roughly larger for large $D$ or $h / D$. For Fr $>1.2$, after air-entrainment, $C_{D}$ values are monotonous and seem to vary linearly 


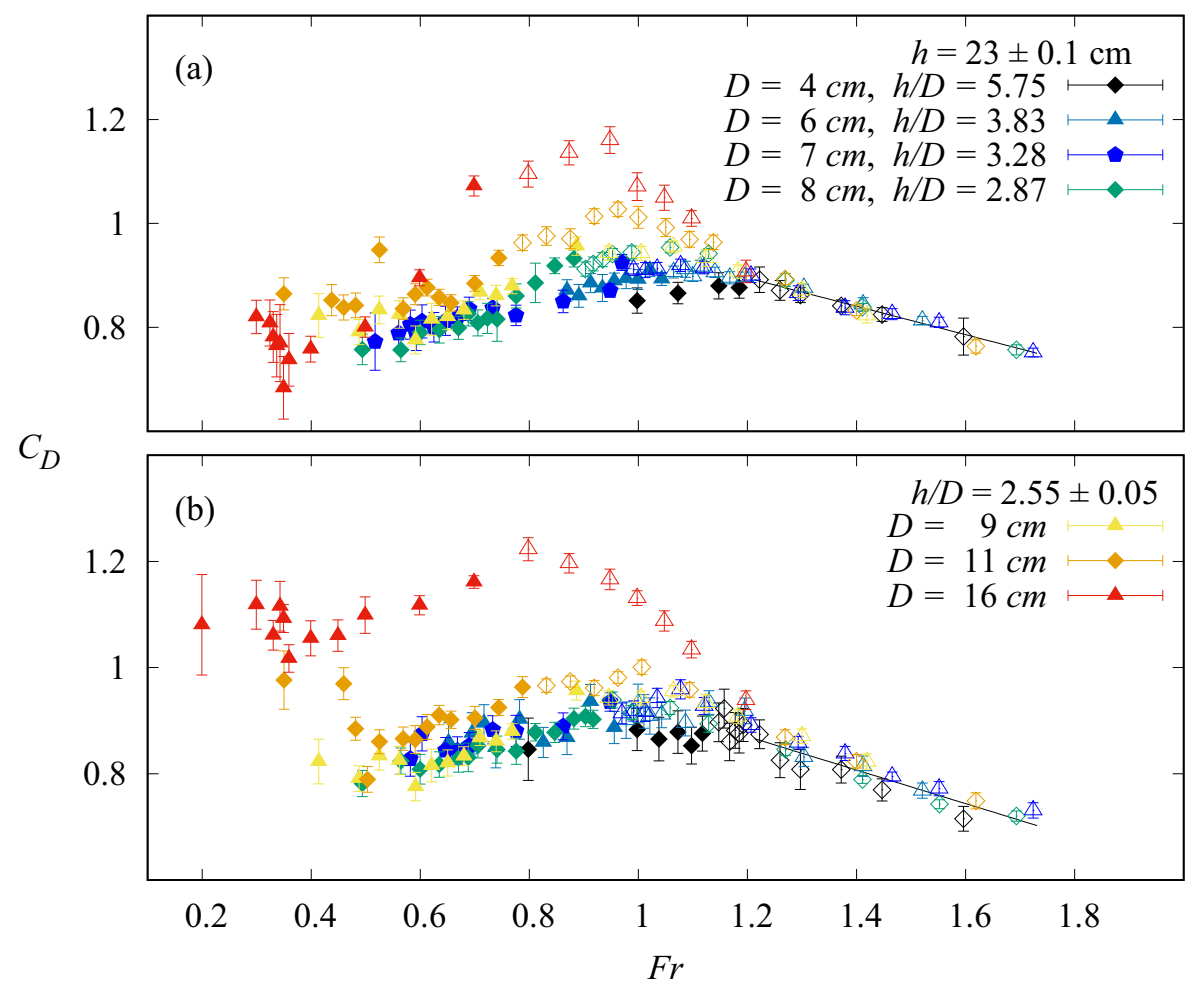

FIG. 4. Drag coefficients, $C_{D}$, as a function of the Froude number, Fr, for different cylinder diameters $D$. Panel (a) corresponds to a constant immersion depth of $h=23 \pm 0.1 \mathrm{~cm}$ and panel (b) corresponds to a constant ratio of $h / D=2.55 \pm 0.05$. Solid symbols represent the situation without air-entrainment and empty symbols represent flow with air-entrainment in the cavity. For $\mathrm{Fr}>1.2, C_{D}$ is linear and the black line is a linear fit.

with Fr. A similar linear behavior has also been observed in granular flows around an immersed cylinder [37].

\section{B. Air-entrainment}

Two mechanisms of air-entrainment are revealed: (i) in the wake thanks to vortices and (ii) in a cavity behind the cylinder. For $1.4<D<4 \mathrm{~cm}(5200<\mathrm{Re} / \mathrm{Fr}<25000)$, air-entrainment occurs in the cavity along the cylinder exclusively. This cavity $[12,13]$ forms viscous cusps where airentrainment is systematically injected. Once air bubbles form below the cavity, they are carried downstream into the wake. The bubbles created by air-entrainment in the cavity have an equivalent diameter ranging from $6 \mathrm{~mm}$ for $D=3 \mathrm{~cm}$ to $8.5 \mathrm{~mm}$ for $D=16 \mathrm{~cm}$.

For $4<D<16 \mathrm{~cm}$, air-entrainment first appears in the wake when Re is increased. Then, as the velocity increases, air-entrainment also appears in the cavity. For a cylinder of $5 \mathrm{~cm}$ in diameter, Fig. 5(a), at $\operatorname{Re}=34670$, shows free-surface deformation without air-entrainment. In Fig. 5(b) at $\operatorname{Re}=39700$, air-entrainment occurs in the cylinder wake. Finally, in Fig. 5(c), at $\operatorname{Re}=45700$, the cavity induces air-entrainment more intensely. For $D=16 \mathrm{~cm}$ and higher Re, the mechanisms of air-entrainment presented in Fig. 6 are similar. Air-entrainment appears in the wake [see Fig. 6(a)] and then in the cavity [see Fig. 6(b)]. The corresponding movies are available as Supplemental Material [38]. 

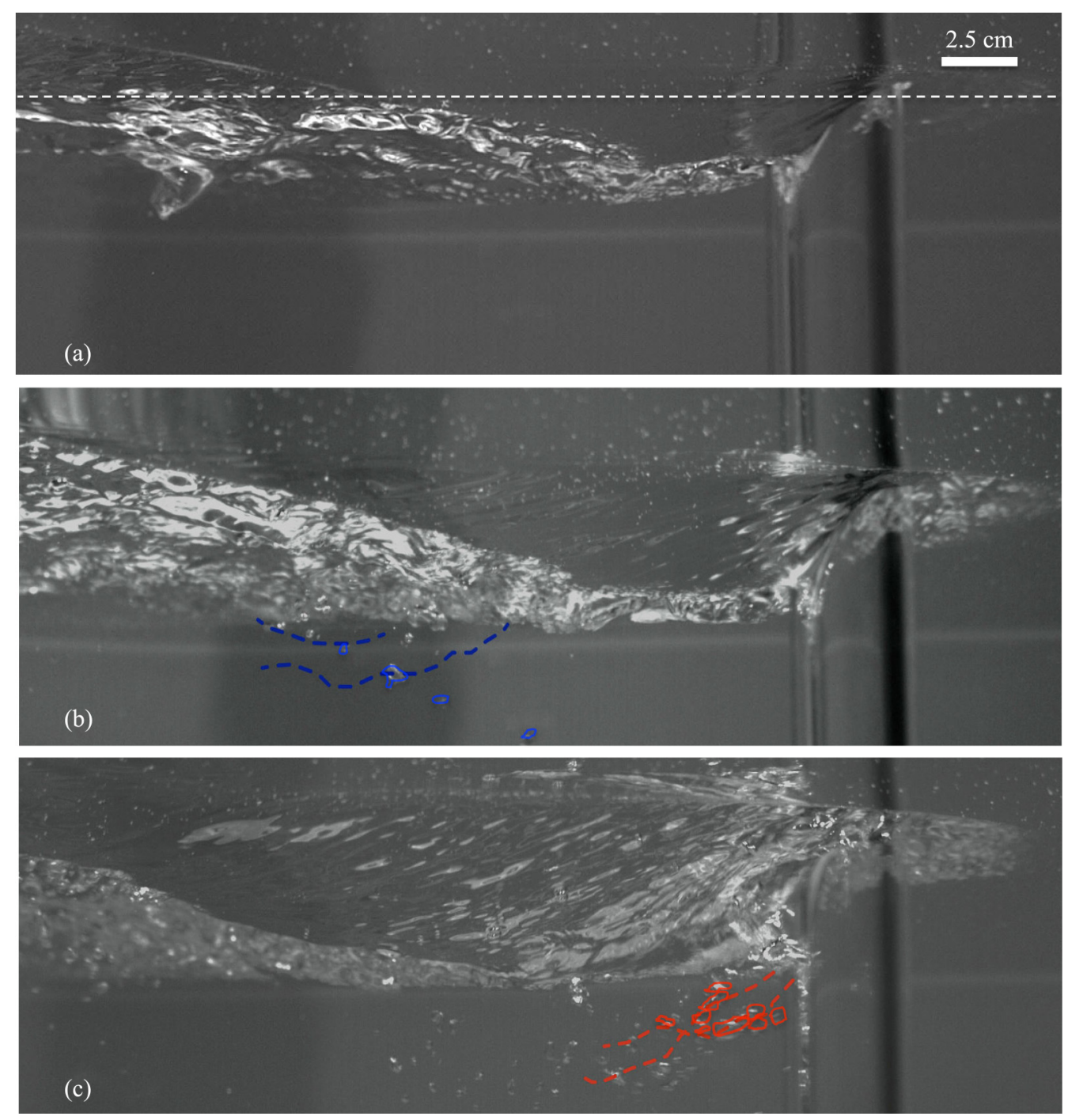

FIG. 5. View from the side below-surface of the cylinder of $5 \mathrm{~cm}$ in diameter. (a) No air-entrainment at $\mathrm{Re}=34670$ or $\mathrm{Fr}=0.97$, see supplementary movie 1 [38]. (b) Air-entrainment in the wake at $\operatorname{Re}=39700$ or $\mathrm{Fr}=1.11$; bubbles encircled in blue, see Supplemental Material, movie 2 [38]. (c) Air-entrainment in the cavity behind the cylinder at $\mathrm{Re}=45680$ or $\mathrm{Fr}=1.3$; bubbles encircled in red, see Supplemental Material, movie 3 [38]. The dashed lines represent bubble trajectories.

A summary of all our observations is presented in Fig. 7, for both constant $h$ and $h / D$. In both cases, several regimes are observed where the different modes of air-entrainment are (i) in the wake, (ii) in the cavity, and (iii) in the wake and the cavity. Boundaries can be drawn between the different regimes and it is the purpose of the model below to explain the nature of some of these boundaries. The main difference between the two diagrams is the area of the wake region, which is wider for large diameters and constant $h$. For small $h / D$, the onset of air-entrainment occurs at smaller Fr presumably because of the size of the recirculation below the free-surface [15,18]. Additionally, the fact that the two diagrams are almost indistinguishable suggests that the immersed height $h$ 

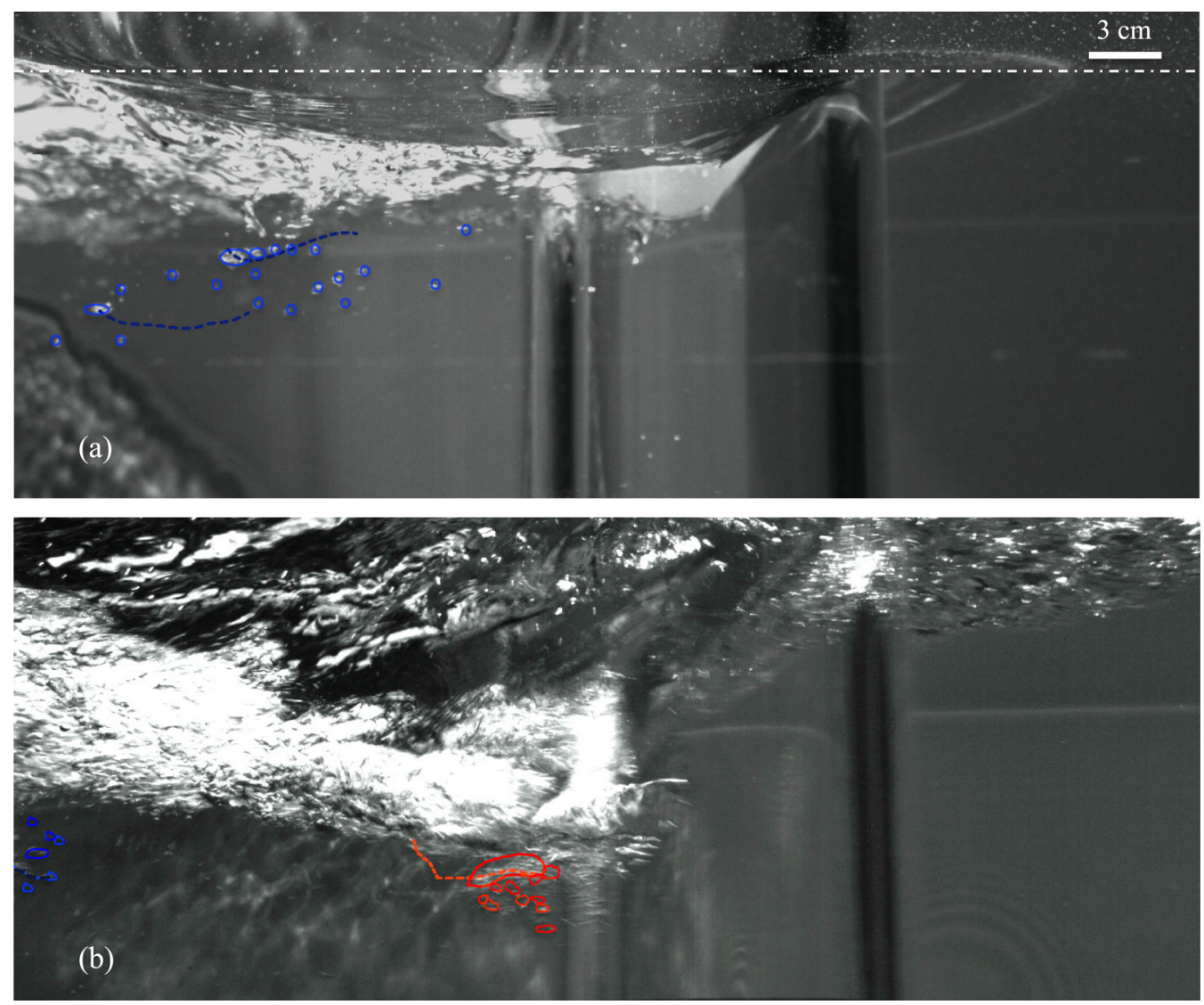

FIG. 6. View from the side below-surface of the cylinder of $16 \mathrm{~cm}$ in diameter. (a) Air-entrainment in the wake at $\mathrm{Re}=122500$ or $\mathrm{Fr}=0.6$; bubbles encircled in blue, see Supplemental Material, movie 4 [38]. (b) Air-entrainment in the cavity behind the cylinder at $\mathrm{Re}=199300$ or $\mathrm{Fr}=1$; bubbles encircled in red, see Supplemental Material, movie 5 [38]. The dashed lines represent observed bubble trajectories.

has little effect on the air-entrainment when $h>23 \pm 0.1 \mathrm{~cm}$ and $h / D>2.55 \pm 0.05$. Hence, airentrainment is mainly due to the free-surface dynamics and deformation.

Indeed, the main feature associated with air-entrainment is the deformation of the cavity behind the cylinder. The optical method described earlier and the direct observations of graduations along the cylinder allowed us to measure the cavity depth $\mathcal{L}$ for various conditions. Specifically, the observations of graduated cylinders are reported in Fig. 8: $\mathcal{L} / D$ as a function of $\mathrm{Fr}^{2} \operatorname{Re} . \mathcal{L}$ represents the mean value from ten snapshots [see Figs. 8(c) and 8(d)]. The error bars are the maximum observed deviation from the mean value. Clearly, the behavior is nonlinear, typically after air-entrainment takes place. However, in the range of $\operatorname{Fr}^{2} \operatorname{Re}$ before air-entrainment, $\mathcal{L} / D$ varies linearly with $\mathrm{Fr}^{2} \mathrm{Re}$. The proportionality coefficients seem to decrease with the diameter. These measurements are insufficient to provide a definite scaling of the cavity height. In fact, the cavity is unsteady and the liquid motion is more disordered as the velocity increases.

Air-entrainment phenomenon in the cavity could be explained using the model developed by Benusiglio [20] where the acceleration of the cavity $\gamma$ is balanced by the gravitational acceleration g. $\gamma$ is controlled by the vortex shedding frequency $f$, calculated from the Strouhal number. In the range of $\mathrm{Re}$ of the present study, St is assumed constant ( 0.2) [5], although some studies have shown its variability upon the cylinder aspect ratio $[18,39,40]$. The acceleration $\gamma$ of the cavity of 

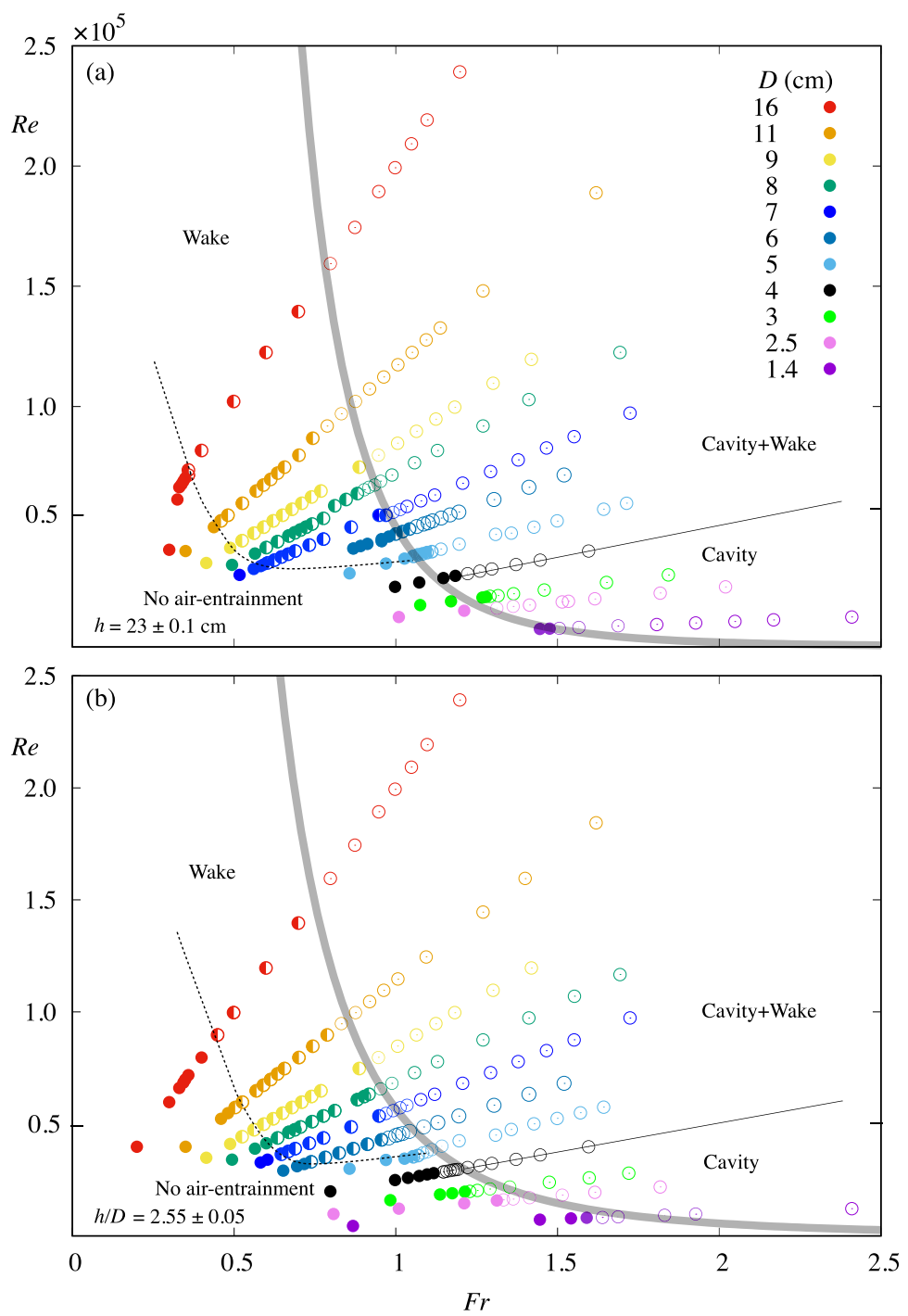

FIG. 7. Flow diagrams Re versus Fr depicting the different air-entrainment regimes: no air-entrainment (solid symbols), air-entrainment in the cavity (partially solid symbols), and air-entrainment in both the cavity and the wake (empty symbols) for different cylinder diameters or Re/Fr. (a) For constant immersion $h=$ $23 \pm 0.1 \mathrm{~cm}$ and (b) for constant $h / D=2.55 \pm 0.05$. The lines are guides to the eyes delimiting the different air-entrainment regimes.

depth $\mathcal{L}$ is defined as follows:

$$
\gamma=\mathcal{L} f^{2}
$$

In Eq. (2), $\mathcal{L}$ is determined using Bernoulli's equation between two positions upstream and downstream of the cylinder. At the upstream position the mean flow velocity is $U$, whereas at the downstream position the flow velocity is taken as the maximum velocity $\mathcal{U}$ of a Rankine vortex $\Gamma / 2 \pi a$ [41], where $\Gamma$ is the circulation around half of the cylinder $D U / 2$ and $a$ represents the core radius of the vortex. The parameter $a$ scales with $D / \sqrt{\mathrm{Re}}$, the boundary layer thickness [42]. With 


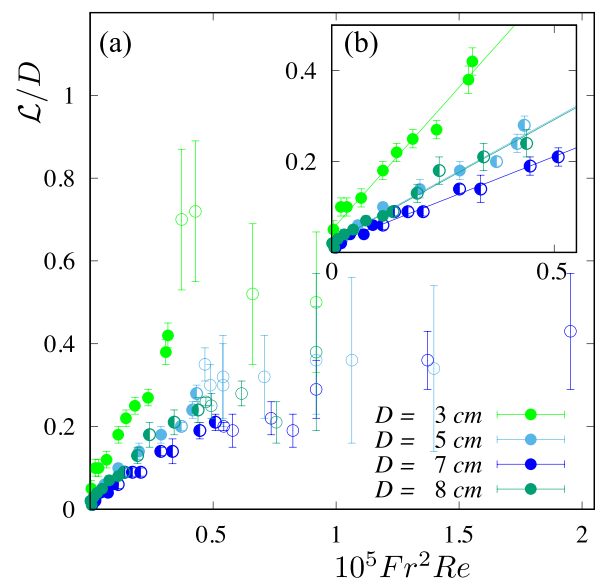

(c)

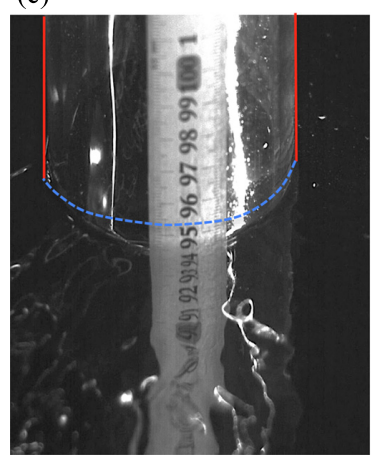

(d)

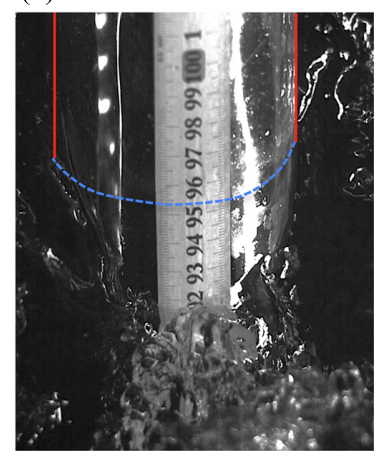

FIG. 8. (a) Nondimensional cavity depth $\mathcal{L} / D$ as a function of $\mathrm{Fr}^{2} \mathrm{Re}$, for different diameters. The inset (b) is a zoom restricted to the regime of no or weak air-entrainment, where the continuous lines are linear fits. Solid symbols represent the situation without air-entrainment and empty symbols represent flow with air-entrainment in the cavity. Panels (c) and (d) are snapshots of the cavity, view from the back, typical of no-air entrainment and air-entrainment, respectively. The continuous vertical red lines represent the emerged cylinder walls and the dashed blue line represents the free-surface at rest.

these assumptions, Benusiglio [20] proposed a scaling for $\mathcal{L}$ :

$$
\mathcal{L} \propto \frac{D U^{3}}{g v},
$$

which has not been validated experimentally or numerically. However, our current measurements (see Fig. 8), using the direct cavity depth observations, seem to be consistent with $\mathcal{L} / D \propto \mathrm{Fr}^{2} \mathrm{Re}$, which is equivalent to Eq. (3). Finally, air-entrainment occurs when $\gamma \simeq g$; therefore Eq. (2) leads to the critical velocity $U_{c}$ for air-entrainment in the cavity $\mathcal{L}$ :

$$
U_{c} \propto\left(\nu g^{2}\right)^{\frac{1}{5}} D^{\frac{1}{5}} \text {. }
$$

Figure 9(a) shows the critical velocity $U_{c}$ for air-entrainment in the cavity $\mathcal{L}$ as a function of $D$ at constant immersion $h=23 \pm 0.1 \mathrm{~cm}$ and constant $h / D=2.55 \pm 0.05$. The error bars of $U_{c}$ correspond to the velocity step between the maximum velocity when air-entrainment is not observed and the velocity where the first bubble appears. The evolution of $U_{c}$ is fitted with power laws of $D^{0.2 \pm 0.02}$ for $h / D \simeq 2.55$ and $D^{0.22 \pm 0.02}$ for $h \simeq 23 \mathrm{~cm}$. Experimental results show good agreements with the theoretical model introduced with Eq. (4). The simple model based on energy conservation with Bernoulli's equation predicts the appearance of air-entrainment in the cavity. Additional data from Benusiglio [20] are also plotted and fit well the present data for $D \leqslant 6 \mathrm{~cm}$. For larger diameters, the critical velocity given by Benusiglio [20] refers to air-entrainment in the cylinder wake like in Fig. 5(b), while our present data refer only to air-entrainment in the cavity. Figure 9(b) presents the evolution of $\operatorname{Re}_{c}$ as a function of $\mathrm{Fr}_{c}$, based on the critical velocity $U_{c}$. The dimensionless form of Eq. (4) is $\operatorname{Re}_{c} \propto \mathrm{Fr}_{c}^{-4}$. The evolution of $\operatorname{Re}_{c}$ is fitted with a power law of $\mathrm{Fr}_{c}^{-3.3 \pm 0.62}$ for $h=23 \pm 0.1 \mathrm{~cm}$ and $\mathrm{Fr}_{c}^{-4.7 \pm 0.51}$ for $h / D=2.55 \pm 0.05$, in good agreement with the prediction of the model. The red shaded area refers to the range of dimensionless parameters where no air-entrainment in the cavity occurs. The black dashed-dotted line represents the threshold $\mathrm{Re} / \mathrm{Fr} \simeq 25000$ above which air-entrainment occurs in the wake and in the cavity. Hence, air-entrainment requires large inertial effects or strong gravity effects. It is interesting to note that the stability diagram in Fig. 9(b) is consistent with the results of Kumagai et al. [43] for a horizontal cylinder translating beneath an air-water interface. 

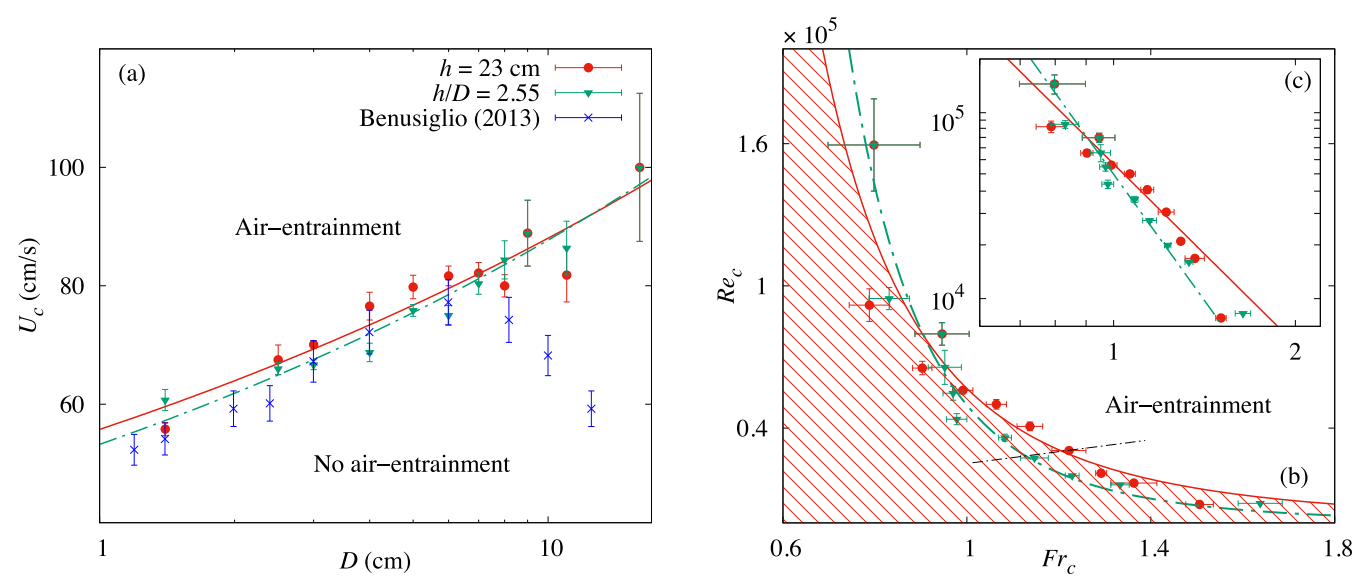

FIG. 9. (a) Critical velocity $U_{c}$ for air-entrainment in the cavity $\mathcal{L}$ as a function of the cylinder diameter $D$ for constant immersion $h=23 \pm 0.1 \mathrm{~cm}$ and constant $h / D \simeq 2.55 \pm 0.05$. Results of Benusiglio [20] are plotted with blue crosses. The green dotted line represents the power law $D^{0.22 \pm 0.02}$ for the constant $h / D$ case and the red solid line represents the power law $D^{0.20 \pm 0.02}$ for $h=23 \pm 0.1 \mathrm{~cm}$. (b) Critical Reynolds number, $\mathrm{Re}_{c}$, as a function of the critical Froude number, $\mathrm{Fr}_{c}$. The black dashed-dotted line represents $\mathrm{Re} / \mathrm{Fr} \simeq 25000$. The shaded region corresponds to no air-entrainment. The inset (c) is the same data in log-log scale.

\section{CONCLUSIONS}

Experimental results on the dynamics of the flow around a vertical translated cylinder have been presented. These deal with the turbulent regime before the so-called fully ventilated regime when air reaches the bottom of the cylinder. There is a critical velocity for the onset of air-entrainement. Then, three modes of air-entrainment are observed depending on the diameter and the translating velocity. For $D \leqslant 4 \mathrm{~cm}$, air-entrainment only occurs in the cavity downstream of the cylinder. For $D \geqslant 5 \mathrm{~cm}$, air-entrainment first appears in the wake and then also in the cavity when Re or Fr is increased. This study focuses on air-entrainment in the cavity, where forces, flow visualization, and critical velocities for air-entrainment are reported at constant $h$ or $h / D . C_{D}$ show an increase of the drag coefficient with Re followed by a decrease after air-entrainment occurred in the cavity. A similar evolution is observed for drag-force measurements versus Fr. Moreover, for Fr $>1.2$, the drags for all diameters overlap and vary linearly with Fr. The threshold velocity for air-entrainment in the cavity scales as $U_{c} \propto D^{0.2}$, i.e., $\operatorname{Re}_{c} \propto \mathrm{Fr}_{c}^{-4}$, which is in good agreement with the scaling of the model of Benusiglio [20]. In the future, the shape of the cavity will be studied in more detail in order to quantify the contribution of wetting properties $[29,44]$ and the capillary-gravity waves.

\section{ACKNOWLEDGMENTS}

The authors acknowledge the financial support of the Normandy Council and the European Union ERDF funding's through the NEPTUNE project. We thank Antoine Bonnesoeur and Claude Houssin for his engineering support and Paul François for his help with the experiments. Our work has also benefited from exchanges with Yuji Tasaka (Hokkaido University) and Jimmy Philip (University of Melbourne).

[1] M. Rabaud and F. Moisy, Ship Wakes: Kelvin or Mach Angle? Phys. Rev. Lett. 110, 214503 (2013).

[2] F. Moisy and M. Rabaud, Mach-like capillary-gravity wakes, Phys. Rev. E 90, 023009 (2014). 
[3] B. Drew, A. R. Plummer, and M. N. Sahinkaya, A review of wave energy converter technology, Proc. Inst. Mech. Eng., Part A 223, 887 (2009).

[4] I. López, J. Andreu, S. Ceballos, I. M. de Alegría, and I. Kortabarria, Review of wave energy technologies and the necessary power-equipment, Renewable Sustainable Energy Rev. 27, 413 (2013).

[5] G. Schewe, On the force fluctuations acting on a circular cylinder in crossflow from subcritical up to transcritical Reynolds numbers, J. Fluid Mech. 133, 265 (1983).

[6] M. Braza, R. Perrin, and Y. Hoarau, Turbulence properties in the cylinder wake at high Reynolds numbers, J. Fluids Struct. 22, 757 (2006).

[7] E. Achenbach and E. Heinecke, On vortex shedding from smooth and rough cylinders in the range of Reynolds numbers $6 \times 10^{3}$ to $5 \times 10^{6}$, J. Fluid Mech. 109, 239 (1981).

[8] M. Breuer, A challenging test case for large eddy simulation: High Reynolds number circular cylinder flow, Int. J. Heat Fluid Flow 21, 648 (2000).

[9] D. J. Tritton, Experiments on the flow past a circular cylinder at low Reynolds numbers, J. Fluid Mech. 6, 547 (1959).

[10] B. M. Sumer and J. Fredsøe, Hydrodynamics around Cylindrical Strucures, Advanced Series on Ocean Engineering Vol. 26 (World Scientific, Singapore, 2006).

[11] T. Kawamura, S. Mayer, A. Garapon, and L. Sørensen, Large eddy simulation of a flow past a free surface piercing circular cylinder, J. Fluids Eng. 124, 91 (2002).

[12] G. Yu, E. J. Avital, and J. J. Williams, Large eddy simulation of flow past free surface piercing circular cylinders, J. Fluids Eng. 130, 101304 (2008).

[13] B. Koo, J. Yang, S. M. Yeon, and F. Stern, Reynolds and Froude number effect on the flow past an interface-piercing circular cylinder, Int. J. Nav. Archit. Ocean Eng. 6, 529 (2014).

[14] D. Sumner, J. L. Heseltine, and O. J. P. Dansereau, Wake structure of a finite circular cylinder of small aspect ratio, Exp. Fluids 37, 720 (2004).

[15] R. T. Gonçalves, G. R. Franzini, G. F. Rosetti, J. R. Meneghini, and A. L. C. Fujarra, Flow around circular cylinders with very low aspect ratio, J. Fluids Struct. 54, 122 (2015).

[16] J. R. Chaplin and P. Teigen, Steady flow past a vertical surface-piercing circular cylinder, J. Fluids Struct. 18, 271 (2003).

[17] T. Ducrocq, L. Cassan, J. Chorda, and H. Roux, Flow and drag force around a free surface piercing cylinder for environmental applications, Environ. Fluid Mech. 17, 629 (2017).

[18] M. A. Benitz, D. W. Carlson, B. Seyed-Aghazadeh, Y. Modarres-Sadeghi, M. A. Lackner, and D. P. Schmidt, CFD simulations and experimental measurements of flow past free-surface piercing, finite length cylinders with varying aspect ratios, Comp. Fluids 136, 247 (2016).

[19] S. J. Keough, I. L. Kermonde, A. Amiet, J. Philip, A. Ooi, J. P. Monty, and B. Anderson, Time resolved measurements of wake characteristics from vertical surface-piercing circular cylinders, in Proceedings of the 20th Australasian Fluid Mechanics Conference, 5-8 December 2016, Perth, Australia (Australasian Fluid Mechanics Society, 2016).

[20] A. Benusiglio, Indiscrétions aux interfaces, Ph.D. thesis, Ecole Polytechnique, 2013.

[21] A. Hay, Flow about semi-submerged cylinders of finite length, Technical Report, Princeton University, 1947.

[22] C. Harwood, Y. Young, and S. L. Ceccio, Ventilated cavities on a surface-piercing hydrofoil at moderate Froude numbers: Cavity formation, elimination and stability, J. Fluid Mech. 800, 5 (2016).

[23] S. A. Mäkiharju, C. Gabillet, B.-G. Paik, N. A. Chang, M. Perlin, and S. L. Ceccio, Time-resolved twodimensional X-ray densitometry of a two-phase flow downstream of a ventilated cavity, Exp. Fluids 54, 1561 (2013).

[24] K. T. Kiger and J. H. Duncan, Air-entrainment mechanisms in plunging jets and breaking waves, Annu. Rev. Fluid Mech. 44, 563 (2012).

[25] D. Chirichella, R. Gomez Ledesma, K. T. Kiger, and J. H. Duncan, Incipient air entrainment in a translating axisymmetric plunging laminar jet, Phys. Fluids 14, 781 (2002).

[26] J. Peixinho, P. Mirbod, and J. F. Morris, Free surface flow between two horizontal concentric cylinders, Eur. Phys. J. E 35, 19 (2012). 
[27] M. Tanaka, G. Girard, R. Davis, A. Peuto, and N. Bignell, Recommended table for the density of water between $0{ }^{\circ} \mathrm{C}$ and $40^{\circ} \mathrm{C}$ based on recent experimental reports, Metrologia 38, 301 (2001).

[28] M. L. Huber, R. A. Perkins, A. Laesecke, D. G. Friend, J. V. Sengers, M. J. Assael, I. N. Metaxa, E. Vogel, R. Mareš, and K. Miyagawa, New international formulation for the viscosity of $\mathrm{H}_{2} \mathrm{O}$, J. Phys. Chem. Ref. Data 38, 101 (2009).

[29] J. M. Aristoff and J. W. M. Bush, Water entry of small hydrophobic spheres, J. Fluid Mech. 619, 45 (2009).

[30] M. D. Griffith, J. Leontini, M. C. Thompson, and K. Hourigan, Vortex shedding and three-dimensional behavior of flow past a cylinder confined in a channel, J. Fluids Struct. 27, 855 (2011).

[31] B. Müller-Graf, Six contributions to the hydrodynamics of high speed marine vehicles, Technical Report No. IO85/87 (Versuchsanstalt für Wasserbau und Schiffbau, Berlin, 1987).

[32] A. L. C. Fujarra, C. P. Pesce, F. Flemming, and C. H. K. Williamson, Vortex-induced vibration of a flexible cantilever, J. Fluids Struct. 15, 651 (2001).

[33] T. Sarpkaya, A critical review of the intrinsic nature of vortex-induced vibrations, J. Fluids Struct. 19, 389 (2004).

[34] C. H. K. Williamson and R. Govardhan, Vortex-induced vibrations, Annu. Rev. Fluid Mech. 36, 413 (2004).

[35] G. R. Franzini, R. T. Goncalves, J. R. Meneghini, and A. L. C. Fujarra, Comparison between force measurements of one and two degrees-of-freedom VIV on cylinder with small and large mass ratio, in Proceedings of the 10th International Conference on Flow-Induced Vibration and Flow-Induced Noise (FIV 2012), 3-6 July 2012, Dublin, p. 561.

[36] G. Gomit, Développement de techniques de mesure de surfaces libres par moyens optiques: Application à l'analyse de l'écoulement généré par un modèle de bateau en bassin des carènes, Ph.D. thesis, Université de Poitiers, 2013.

[37] D. Chehata, R. Zenit, and C. R. Wassgren, Dense granular flow around an immersed cylinder, Phys. Fluids 15, 1622 (2003).

[38] See Supplemental Material at http://link.aps.org/supplemental/10.1103/PhysRevFluids.4.064801 for movies of the flow and air-entrainment behind the cylinder of Figs. 5 and 6.

[39] D. J. Farivar, Turbulent uniform flow around cylinders of finite length, AIAA J. 19, 275 (1981).

[40] T. A. Fox and G. S. West, Fluid-induced loading of cantilevered circular cylinders in a low-turbulence uniform flow. Part 1: Mean loading with aspect ratios in the range 4 to 30, J. Fluids Struct. 7, 1 (1993).

[41] H.-F. Li, H.-X. Chen, M. A. Zheng, and Z. Yi, Experimental and numerical investigation of free surface vortex, J. Hydrodyn., Ser. B 20, 485 (2008).

[42] H. Schlichting, K. Gersten, E. Krause, H. Oertel, and K. Mayes, Boundary-Layer Theory (Springer, Berlin, 1955).

[43] I. Kumagai, Y. Murai, Y. Tasaka, and N. Nakamura, Bubble generation by a cylinder moving beneath a free surface, J. Fluid Sci. Technol. 6, 851 (2011).

[44] T. T. Truscott, B. P. Epps, and J. Belden, Water entry of projectiles, Annu. Rev. Fluid Mech. 46, 355 (2014). 